\title{
ANÁLISIS DE LOS EPISODIOS DE LLUVIA TORRENCIAL EN EL PARQUE NACIONAL DE LA CALDERA DE TABURIENTE (LA PALMA, ISLAS CANARIAS, ESPAÑA)
}

\author{
Pablo MÁYER SUÁREZ1 ${ }^{1}$, María Victoria MARZOL JAÉN², \\ José HERIBERTO LORENZO ${ }^{3}$, Andrés DÍEZ-HERREO ${ }^{4}$, Mar GÉNOVA FUSTER ${ }^{5}$, \\ Miguel Ángel SAZ SÁNCHEZ ${ }^{6}$ \\ ${ }^{1}$ Instituto de Oceanografia y Cambio Global (IOCAG), ULPGC. \\ ${ }^{2}$ Departamento de Geografia e Historia, ULL. \\ ${ }^{3}$ Parque Nacional de la Caldera de Taburiente. \\ ${ }^{4}$ Instituto Geológico y Minero de España. \\ ${ }^{5}$ Escuela Universitaria de Ingeniería Técnica Forestal, UPM. \\ ${ }^{6}$ Departamento de Geografia, Universidad de Zaragoza. \\ pablo.mayer@ulpgc.es, mmarzol@ull.es, caldera.cmayot@gobiernodecanarias.org, \\ andres.diez@igme.es, mar.genova@,upm.es, masaz@unisaz.es
}

\section{RESUMEN}

El Parque Nacional de la Caldera de Taburiente en la isla de La Palma, con un promedio anual de 390.000 visitantes en los últimos 5 años, tiene en los procesos de vertientes y las avenidas torrenciales sus dos principales amenazas. El objetivo de este trabajo es analizar, mediante la única serie de precipitación disponible en el entorno del Parque Nacional, las características de la pluviosidad y de los episodios de lluvia extrema ( $\geq 200 \mathrm{~mm} / 24 \mathrm{~h}$ ) ocurridos entre 1978 y 2013. Se determinan sus causas atmosféricas, su distribución espacial y las consecuencias geomorfológicas y antrópicas que tienen en el ámbito de la Caldera. En este entorno la precipitación tiene una fuerte concentración, pues a) el $80 \%$ de la misma se produce en el $25 \%$ de los días más 11 viosos, b) el 50\% de los episodios lluviosos se registran en un único día y c) en el $45 \%$ de los días más lluviosos la intensidad es igual o superior a ese umbral. La principal causa de esos episodios es la presencia de DANAS en los niveles medios y altos de la troposfera, observándose diferencias según la procedencia de las perturbaciones en superficie o la existencia, incluso, de situaciones anticiclónicas. Estos episodios causan significativas modificaciones en los cauces y vertientes por la movilización de importantes volúmenes de sedimentos $\mathrm{y}$, sobre todo en las últimas décadas, víctimas mortales, generalmente turistas, que son arrastrados por súbitas avenidas o que tienen que ser evacuados al quedar aislados en el interior del Parque.

Palabras clave: Parque Nacional, Taburiente, episodios de lluvia extrema, riesgo, avenidas torrenciales.

\begin{abstract}
The Caldera de Taburiente National Park on the island of La Palma has had an average of 390,000 visitors a year over the past 5 years. The greatest threat to this park is when extreme heavy rainfall comes down the steep slopes and sweeps away rocks and anything else in its path. The aim of this paper is to analyze, using the only
\end{abstract}


series of rainfall available for the National Park, the characteristics of rainfall and episodes of extreme rainfall $(\geq 200 \mathrm{~mm} / 24 \mathrm{~h})$ which occurred between 1978 and 2013 . The atmospheric causes and the spatial distribution of the episodes of extreme rainfall and the geomorphological and anthropical consequences on the environment of the Caldera are determined. The precipitation in this environment is highly concentrated, because a) $80 \%$ of it occurs on $25 \%$ of the rainiest days, b) $50 \%$ of the rainy episodes are recorded in a single day and c) on $45 \%$ of the rainiest days, the intensity is equal to or greater than the abovementioned threshold. The main cause of these episodes is the presence of DANAS in the middle and upper levels of the troposphere, with differences being observed depending on the origin of low-pressure area on the surface or even the existence of anticyclone situations. These episodes cause significant changes in the water courses and slopes by the movement of significant volumes of sediment and above all have caused human deaths, usually of tourists, who are carried away by sudden floods or who have had to be evacuated when they have been stranded in the interior of the park.

Key words: National Park, Taburiente, risk, episodes of extreme rainfall, flash flood.

\section{INTRODUCCIÓN}

El Parque Nacional de la Caldera de Taburiente ocupa una posición central en la isla canaria de La Palma. Tiene una superficie de 4.690 hectáreas, $8 \mathrm{~km}$ de diámetro, desniveles de más de 2.000 metros (el pico más alto situado en el sector septentrional de la caldera es el Roque de los Muchachos con $2.426 \mathrm{~m}$ de altitud) y su perímetro es de $27 \mathrm{~km}$. Fue declarado como tal el 6 de octubre de 1954 y en su interior alberga una zona especial de protección de aves (ZEPA), un lugar de interés comunitario (LIC) y es, junto con el resto de la isla, Reserva de la Biosfera desde el año 2002. El promedio de visitantes entre 2006 y 2014 es de 382.447 y en 2014 visitaron el parque 382.447 personas.

El motivo de su declaración como Parque Nacional es su alto valor geológico (BOE $n^{\circ} 303,30 / 10 / 1954$, p.7292) y en la actualidad es el principal atractivo turístico. El término que le da nombre proviene de una de las depresiones más grandes existentes en el mundo en su género: una caldera erosiva escavada sobre el edificio volcánico más antiguo de la isla (Rodríguez-Fernández, 2011). Hay que señalar que las imponentes pendientes de la Caldera, la alternancia de materiales de distinta naturaleza (volcánica, subvolcánica y plutónica) muchos de ellos deleznables, y los fuertes aguaceros que se producen en su interior motiva la generación de movimientos en masa, incorporando materiales detríticos de diverso tamaño a las aguas de escorrentía. Esta dinámica ha sido estudiada por Díez-Herrero et al. (2012) y Génova et al. (2015) utilizando técnicas como la dendrogeomorfología que ha permitido datar, junto con los escasos datos de precipitaciones disponibles, los principales eventos de lluvia torrencial en la Playa de Taburiente, uno de los lugares más transitados y uno de los principales accesos al área de servicios y zona de acampada del espacio protegido

Es necesario reseñar que entre los motivos que justifican su declaración como Parque Nacional se alude a "aguas cayendo impetuosas en grandes trombas invernales" (BOE, nº 303, 30/10/1954). Es, precisamente, el análisis de la precipitación como 
factor de peligrosidad lo que este trabajo pretende profundizar al tiempo que se caracterizan los principales eventos tormentosos, se identifican sus causas atmosféricas y se sintetizan sus consecuencias socioterritoriales.

\section{LOS DATOS Y EL MÉTODO}

En el interior de La Caldera y el entorno del Parque Nacional sólo hay 5 pluviómetros, gestionados por la Agencia Estatal de Meteorología -AEMET- (figura 1). De ellos, sólo uno (C106U-Caldera de Taburiente-Taburiente) posee datos diarios. Sin embargo, se trata de una serie irregular pues comienza en octubre de 1957, se interrumpe desde noviembre de 1958 a junio de 1973; desde ésta fecha hasta septiembre de 1977 sólo registra datos durante 13 meses de manera discontinua. A partir de 1978 la serie pluviométrica adquiere cierta continuidad, aunque con ausencia de datos entre octubre de 1981 y agosto de 1984 y meses aislados entre 1998 y 2008. Así pues, sólo se dispone de 22 años con información completa. Los restantes pluviómetros son totalizadores mensuales cuyos registros comienzan en la década de 1980, pero sus series tampoco son continuas.

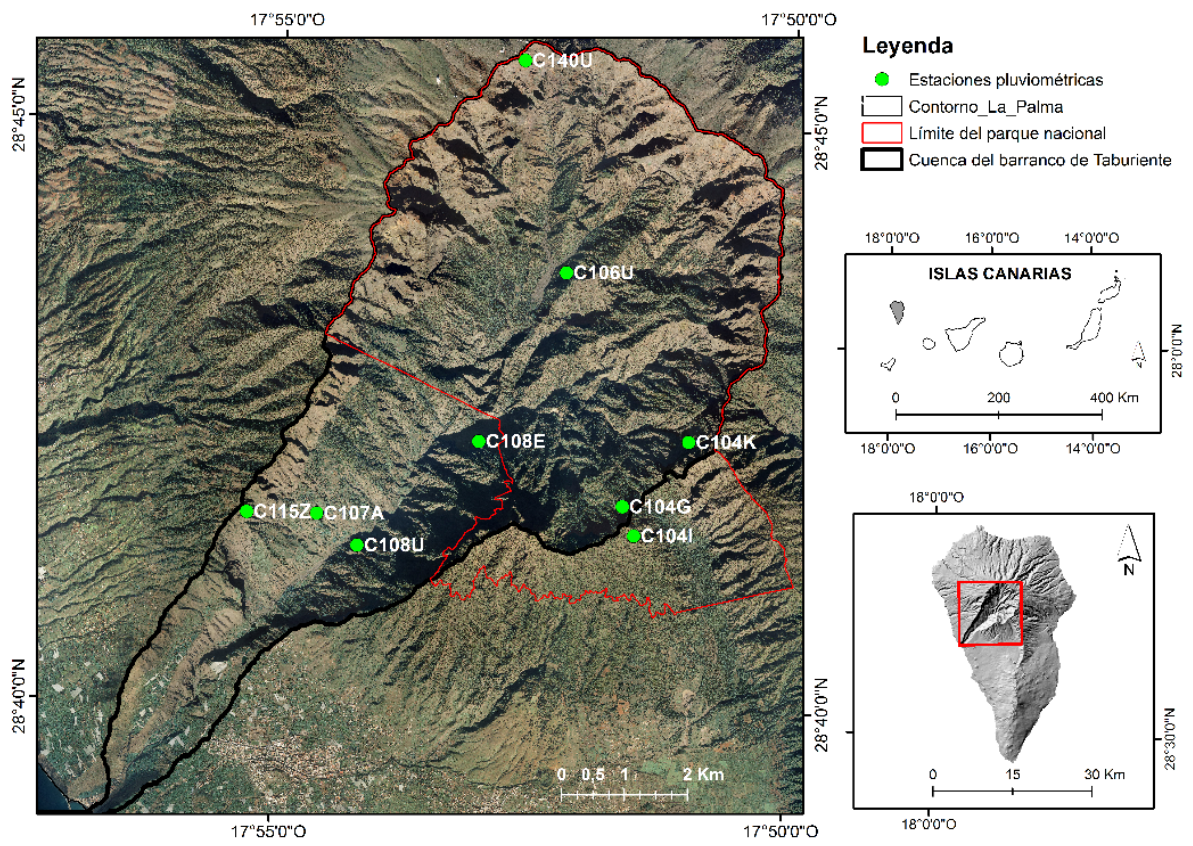

Fig. 1: Localización de las estaciones pluviométricas en la cuenca del barranco de Taburiente. Fuente: de las estaciones pluviométricas AEMET; de la ortofoto digital: Grafcan, SA.

Dado que el objetivo principal es establecer la peligrosidad de la pluviosidad la metodología empleada es la analítica, de tipo estadístico, aplicada a los datos diarios del período 1978 a 2013, de la estación C106U situada prácticamente en el centro de la cuenca a 820 metros de altitud. 
El trabajo se ha realizado a distintas escalas temporales. Por un lado, se analizan las variaciones interanuales de la precipitación aplicando estadísticos básicos como la media, la desviación tipo y el coeficiente de variación. Un segundo análisis, a escala mensual, permite conocer los meses con mayor concentración de la precipitación y caracterizar su torrencialidad. Finalmente, del análisis diario se infiere cuestiones referentes a la duración de la lluvia, su concentración y los episodios más intensos registrados durante el período de estudio.

Una vez identificados los episodios de lluvia torrencial, utilizando el umbral de $200 \mathrm{~mm} / 24$ horas, se analizan las situaciones atmosféricas mediante la consulta de los mapas sinópticos de superficie y $300 \mathrm{hPa}$ disponibles en los Boletines Meteorológicos Diarios de la AEMET.

Finalmente, para el análisis de las consecuencias socioeconómicas y territoriales se consultan los documentos editados por el antiguo Instituto Nacional de Conservación de la Naturaleza (Arozarena et al., 1976) así como la prensa escrita (Diario de Avisos, El Día, Canarias 7 y La Provincia). La Universidad de Las Palmas de Gran Canaria tiene disponible en la web de su biblioteca una base de datos (Jable) donde se encuentran digitalizada la prensa escrita de Canarias. Este recurso ha permitido, mediante búsquedas selectivas, identificar las noticias de interés desde 1950 hasta la actualidad.

\section{RESULTADOS}

\subsection{La lluvia anual y mensual}

La posición favorable del sector occidental de la isla de La Palma a la llegada de frentes y perturbaciones atlánticas implica unas cantidades considerables de precipitación anual. La media anual es de 1.016,8 mm, que caen en tan sólo 35 días de lluvia lo que supone que sólo llueve algo menos del 10\% de los días del año.

Uno de los rasgos que definen la precipitación en Canarias es su irregularidad (Marzol et al., 2006). Aunque este rasgo es mucho más evidente en las zonas orientales y meridionales próximos a la costa, con coeficientes de variación que superan el 70\% (Dorta, 2007); no menos significativo es este índice en las zonas de altitud media orientadas al Oeste, así como las zonas de cumbre. Taburiente, que se encuentra en el primer ámbito, posee un coeficiente de variación del 50\%. Otros índices que corroboran esa irregularidad pluviométrica es el valor acusado de la desviación tipo, $526,8 \mathrm{~mm}$. Tal y como señalan Marzol et al. (2006), las precipitaciones en las islas atlánticas de la Macaronesia tienen un fuerte carácter estacional y los valores de la serie de Taburiente así lo demuestran. Más de la mitad de la lluvia cae en invierno, el $56 \%$, en otoño un $25 \%$ y en la primavera un $19 \%$. Durante el verano, meses en los que las islas están bajo la acción del anticiclón de las Azores, las cantidades recogidas son poco significativas y sólo representan un $1 \%$. Los meses más lluviosos son diciembre y enero, en los que se recoge, de media, $227,1 \mathrm{~mm}$ y $213,3 \mathrm{~mm}$ respectivamente; ello supone que más del $40 \%$ de la lluvia anual cae sólo en los dos meses más lluviosos. No obstante, no es difícil encontrar meses en los que se triplican esas cantidades como diciembre de 2009 (703,2 mm) o enero de 1980 (556,5 mm). Como caso extremo destacan las lluvias registradas durante febrero de 1979, fecha que septuplica la media pues se totalizó la cifra de 1.626,0 mm. 


\subsection{La lluvia diaria}

El análisis de la intensidad diaria de la lluvia en el interior de la Caldera de Taburiente muestra que algo más de un tercio de la precipitación suele tener una intensidad entre débil o muy débil (inferior a 5,0 mm), mientras que aquellas que superan los 100 $\mathrm{mm} / 24$ horas suponen el $5 \%$ de los días de lluvia. Se trata de un porcentaje relativamente elevado y desde el punto de vista de su peligrosidad deben ser considerados por los gestores del Parque. Un índice que permite incidir aún más en la peligrosidad de las lluvias es el Índice de Concentración de las Precipitaciones (Martín-Vide, 2004) que, en el caso de la serie de Taburiente, adquiere el valor de 0,7. Ello implica que el $80 \%$ de la lluvia anual proviene del $25 \%$ de los días más lluviosos. Con respecto a la duración de la lluvia, hay una probabilidad de algo más del $50 \%$ de que ésta caiga en un solo día. Las secuencias lluviosas de dos días suponen el $20 \%$, las de tres días el $10 \%$ y las de cuatro días el 6\%. La precipitación acumulada en todas esas secuencias es muy similar y oscila entre el 14\% y el 9\% de la lluvia anual (figura 2). El período más largo de días consecutivos lloviendo sucedió entre el 12 y el 27 de enero de 1979 pues en esos 6 días se totalizó 1.108,9 mm. Entre ellos habría que señalar los días 17, 23 y 26 con 185,5 mm, 210,0 mm y 197,5 mm respectivamente.

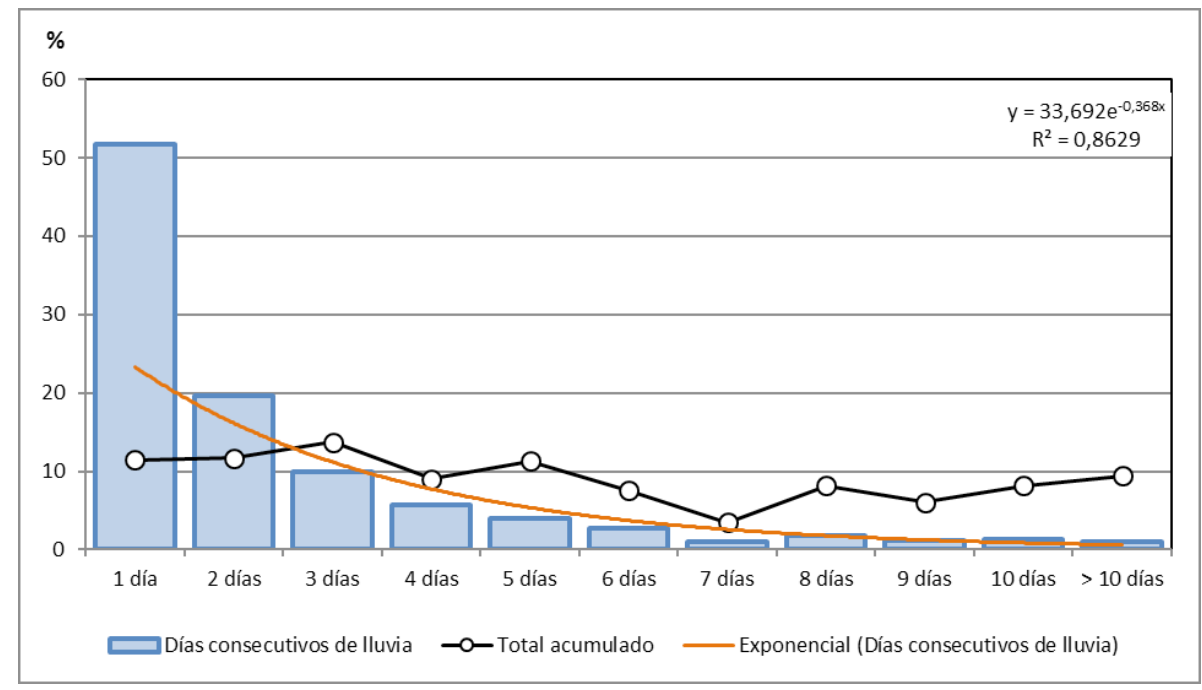

Fig.2: Relación entre la duración de los episodios lluviosos y la cantidad de lluvia recogida en la Caldera de Taburiente. Fuente: AEMET.

Existe una probabilidad del $30 \%$ de que diciembre registre el día más lluvioso del año. Esa probabilidad se reduce casi a la mitad (el 18\%) en los meses de enero, febrero y octubre. Con respecto a la cuantía de agua que cae el día más lluvioso del año lo más habitual es que ésta tenga una intensidad superior a 100,0 mm en 24 horas. Sin embargo, es preciso destacar el elevado número de días en los que se han superado los $200 \mathrm{~mm} / 24$ horas, pues supone el $45 \%$ de los días más lluviosos. Estas precipitaciones torrenciales son las que generan graves procesos erosivos, así como cotas de inundación muy significativas. 
Pese a las limitaciones de los datos meteorológicos en el ámbito de la Caldera de Taburiente se han podido identificar 11 episodios en los que se alcanzaron o superaron los $200 \mathrm{~mm} / 24$ horas entre 1979 y 2013 (tabla 1). No obstante, hay que señalar que en los años en los que la estación ha estado registrando datos en 54 ocasiones se han superado los $100 \mathrm{~mm} / 24$ horas que, igualmente, pueden producir importantes daños en la cuenca.

\begin{tabular}{|c|c|c|c|c|c|}
\hline $\mathrm{N}^{\mathrm{o}}$ & Fecha & Precipitación $(\mathrm{mm})$ & $\mathrm{N}^{\mathrm{o}}$ & Fecha & Precipitación $(\mathrm{mm})$ \\
\hline 1 & $10 / 02 / 1978$ & 399,3 & 7 & $22 / 12 / 2000$ & 209,5 \\
\hline 2 & $23 / 01 / 1 / 979$ & 210,0 & 8 & $13 / 03 / 2001$ & 200,0 \\
\hline 3 & $25 / 01 / 1980$ & 243,9 & 9 & $19 / 03 / 2007$ & 279,0 \\
\hline 4 & $27 / 02 / 1986$ & 217,0 & 10 & $23 / 12 / 2009$ & 226,0 \\
\hline 5 & $23 / 01 / 1987$ & 240,0 & 11 & $15 / 02 / 2010$ & 241,1 \\
\hline 6 & $04 / 12 / 1991$ & 208,0 & \multicolumn{4}{|l}{} \\
\hline
\end{tabular}

Tabla 1: Precipitaciones $\geq 200 \mathrm{~mm} / 24$ horas en Caldera de Taburiente. Fuente: AEMET.

\subsection{Causas meteorológicas de los episodios de lluvia extrema}

En todos los casos analizados las precipitaciones fueron originadas por la presencia de depresiones aisladas en los niveles altos (DANAS). De los once episodios, ocho (los numerados en la tabla 1 como 1, 2, 3, 5, 6, 7, 10 y 11) se caracterizan por una circulación meandriforme de la corriente en chorro que termina por aislar, en el entorno de Azores y NW de Canarias, un embolsamiento de aire frío. Se genera, en las capas medias y altas de la atmósfera, un eje NW-SE desde la península de Terranova hasta el NW de Canarias que refleja la advección de aire frío en esos niveles. En superficie las borrascas siguen trayectorias W-E, al Sur del paralelo $40^{\circ} \mathrm{N}$, cuyos activos frentes atraviesan la isla generando aguaceros que se ven potenciados por un relieve de acusadas pendientes. En la figura 3 (A1 y A2) se muestra un ejemplo de este tipo de configuración isobárica asociada al episodio de1 23 de enero de 1987 que dejó cantidades de lluvia que superaron los $250 \mathrm{~mm}$ en la cabecera del barranco de Taburiente.

Un matiz diferenciador en cuanto a la advección de la masa de aire frío en los niveles altos y la trayectoria de la perturbación en superficie se corresponde con el episodio del 27 de febrero de 1986 (episodio número 4 de la tabla 1), pues en este caso la masa de aire frío en los niveles altos tiene un marcado eje N-S, desde las islas Británicas y N de la Península Ibérica hasta Canarias. En superficie, la formación de una profunda depresión $(995 \mathrm{hPa})$ que termina situándose al $\mathrm{N}$ de Canarias es fiel reflejo de esta acusada inestabilidad (figura 3B1 y 3B2). En este caso las lluvias más intensas se concentran en el interior de la Caldera con cantidades que superan los $200 \mathrm{~mm}$.

Finalmente, se señala la existencia de situaciones sinópticas que difieren considerablemente de las anteriores, pues se trata de dos episodios en los se forma una depresión aislada en los niveles altos, acompañada de altas presiones en superficie (números 8 y 9 de la tabla 1). Ejemplo de ello fueron las lluvias del 17 de marzo de 2007, que dejaron cantidades de más de 270,0 mm en el interior de la Caldera. En la imagen 3 (C1 y C2) se han insertado los mapas del tiempo del día 19 que muestran de una manera nítida la situación de inestabilidad en las capas altas. 


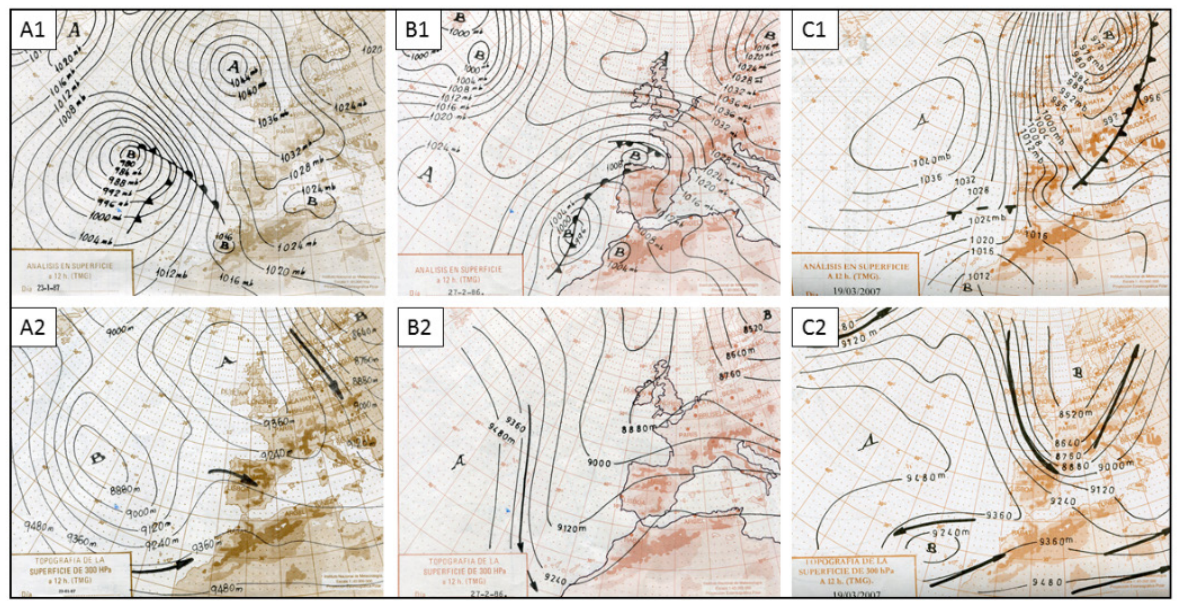

Fig. 3: Mapas del tiempo en superficie y $300 \mathrm{hPa}$ de los episodios de lluvias torrenciales de los días 23 de enero de 1987(A1 y A2), 27 de febrero de 1986 (B1 y B2) y del 19 de marzo de 2007(C1 y C2). Fuente: AEMET.

\subsection{Consecuencias territoriales de los episodios de lluvia torrencial}

De la consulta de la prensa escrita regional, entre 1950 y 2015, se obtienen veinte noticias que hacen referencia a las consecuencias de las lluvias en la cuenca del barranco de Las Angustias. De éstas, ocho sólo aluden al abundante caudal que circula por el cauce, mientras que las doce noticias restantes mencionan diferentes perjuicios. Como es lógico, casi todas las referencias se concentran en la desembocadura del barranco, pues es donde se encuentra el núcleo de población de Los Llanos de Aridane y es donde se han producido las mayores transformaciones en el cauce por la construcción de vías y edificaciones.

Las descripciones de las avenidas hacen referencia a la gran cantidad de sedimentos y restos vegetales que circulan por los diferentes barrancos. Es significativo, por ejemplo, que tras la avenida del 22 de octubre de 1962 se mencione cómo los troncos de los árboles (Pinus canariensis) destrozaron los muros de los edificios cercanos al cauce, además de su inundación entre los que se incluye un centro escolar donde la altura del agua alcanzó un metro.

En lo que concierne a los daños, se mencionan de manera frecuente los problemas de los diques construidos entre los núcleos del Puerto y Tazacorte, y la destrucción de la carretera y el puente que conecta ambas poblaciones. En el periodo analizado al menos en cinco ocasiones se refieren a estos problemas por lo que no es de extrañar que el alcalde de Tazacorte instara, en noviembre de 1987 (Canarias 7, p. 48), a la rápida restauración del muro de contención, entre la iglesia y el mar, por el peligro de derrumbe inminente y la consecuente interrupción del tráfico entre el municipio de Tijarafe y el Puerto, señalando el riesgo que supondría la apertura de un nuevo cauce que terminaría afectando a los habitantes de este último barrio. Esta situación se volvió a repetir en diciembre de 2009, con idénticas consecuencias. 
Otros problemas tienen que ver con el sector agrícola, pues las avenidas torrenciales deterioran los canales que derivan las aguas del cauce hacia los predios agrícolas destruyendo, al tiempo, los cultivos de plataneras más próximos al cauce.

En lo que concierne a las consecuencias en el interior del Parque Nacional de Taburiente destaca, por un lado, la muerte de tres excursionistas que se vieron sorprendidos por las aguas de avenida el 20 de noviembre de 2001. En el momento de las lluvias se encontraban en el interior del Parque 191 personas, que tuvieron que ser evacuadas. En sus relatos cuentan que se vieron sorprendidos por la riada y mencionan cómo el nivel del agua subió rápidamente y trece de ellos pudieron escapar a través de uno de los senderos más próximos. El aislamiento de los excursionistas y su necesaria evacuación es frecuente durante estos episodios de lluvia torrencial, como sucediera el 21 de octubre de 2011 que hubo que evacuar a 52 turistas que quedaron atrapados en el interior del Parque.

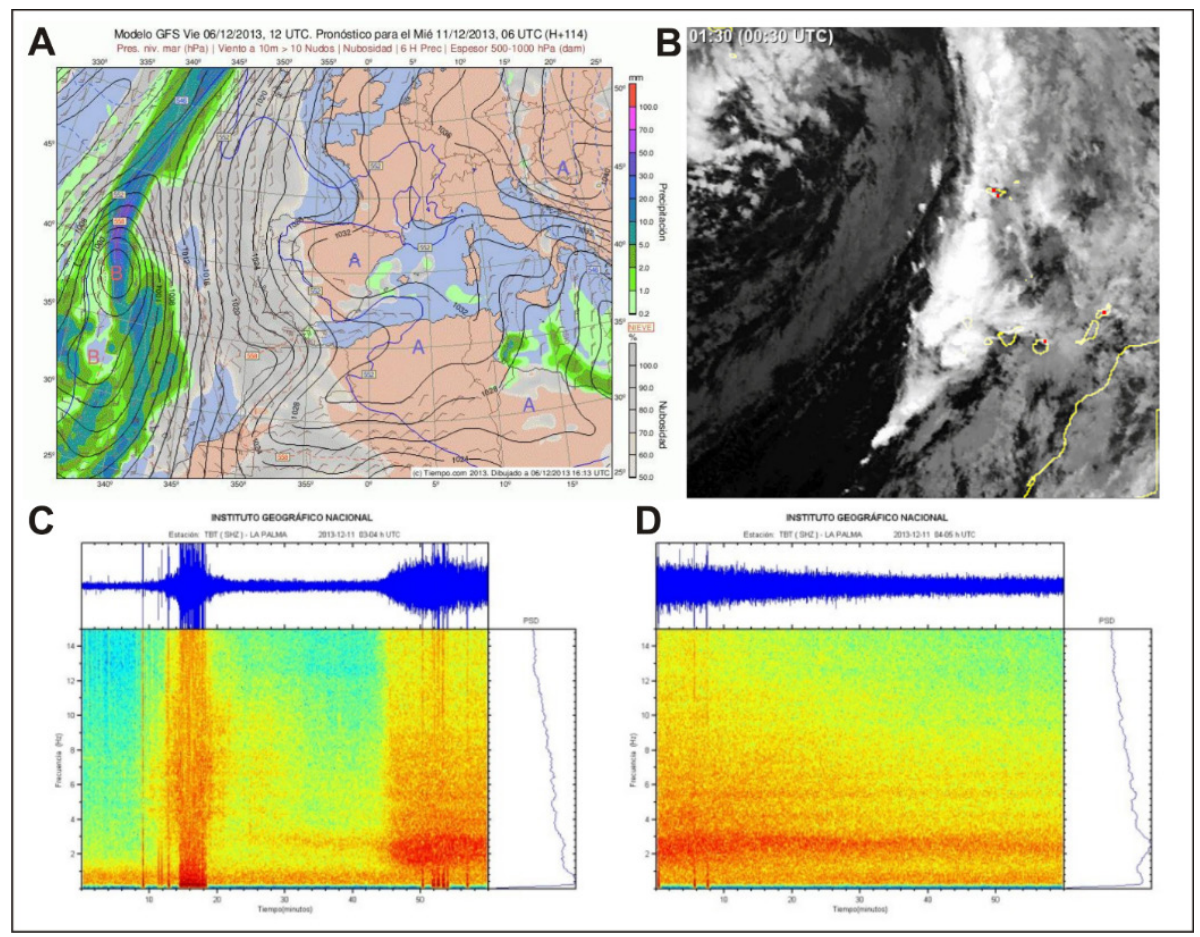

Figura 4. Secuencia de imágenes del episodio torrencial del 11 de diciembre de 2013.A) Modelo de predicción meteorológica GFS previsto para las 12 horas (UTC); B) Imagen del satélite METEOSAT a las 00:30 horas (UTC). Debajo, C) y D), se muestra la secuencia de los espectrogramas horarios de la estación sísmica TBT entre las 04 y las 06 horas UTC. Fuente: www.ogimet.com, Meteosat e IGN.

Finalmente hay que mencionar las profundas transformaciones geomorfológicas que se producen en los diferentes cauces de los barrancos de Taburiente. A raíz de las lluvias intensas del 11 de diciembre de 2013 se dispone de un detallado informe 
realizado por Lorenzo (2013) que relata, de manera pormenorizada, tanto sus causas atmosféricas como sus consecuencias geomorfológicas. Hay que señalar que este episodio de lluvias no alcanzó, en la estación C106U, los $200 \mathrm{~mm} / 24 \mathrm{~h}$, lo que evidencia su carácter local (se concentró en la cara $\mathrm{N}$ del pico Benjenado, al $\mathrm{S}$ de la estación), motivo por el cual no aparece en la tabla 1. Como se observa en la figura 3A, las fuertes precipitaciones obedecen a una depresión que se situó al Noroeste de las islas Canarias, muy similar al episodio del 23 de enero de 1987 (figura 3). Como se puede observar en la imagen del satélite Meteosat (figura 4B) el frente frío comenzó a atravesar la isla de La Palma en la madrugada del día 11, con diversos núcleos convectivos. Los truenos asociados a los rayos generaron importantes vibraciones que quedaron registrados en los sismogramas y espectrogramas de la estación TBT que tiene el IGN en Taburiente (figura 4C y 4D). De la misma manera, también quedó registro de la importante avenida que se produjo durante la tormenta con una señal similar a la de las erupciones volcánicas (tremor). Las ondas sísmicas producidas por los truenos de la tormenta, unido a la fuerte precipitación, produjeron múltiples desprendimientos y flujos de lodo y piedras que modificaron sustancialmente numerosos cauces.

\section{DISCUSIÓN Y CONCLUSIONES}

Hay que señalar las dificultades a la hora de evaluar los daños ocasionados en el entorno del Parque en los diferentes episodios de lluvia, pues las fuentes de documentación se circunscriben a las zonas habitadas y, por tanto, a la desembocadura del barranco, lugar donde se constata la destrucción de vías de acceso, inundaciones y perjuicios en los cultivos situados en las márgenes de los cauces. Sería interesante, para hacer una mejor valoración de los perjuicios producidos en los diferentes eventos, profundizar en otras fuentes de información histórica como, por ejemplo, los documentos donde se alude a las reparaciones de los canales de distribución de agua de las heredades o a los archivos parroquiales y otros documentos históricos del Ayuntameinto de Los Llanos de Aridane. De la misma manera hay que resaltar la utilidad de los análisis dendrogeomorfológicos para, entre otras cuestiones, datar las avenidas torrenciales en áreas como ésta carente de registro de precipitación continua o con series muy cortas (Génova et al., 2015).

Como conclusión se puede indicar que las características geológicas/geomorfológicas de este Parque Nacional motivan una importante dinámica erosiva, especialmente durante los episodios de lluvia intensa. Las profundas transformaciones de los cauces y de las vertientes después de estos episodios suponen, junto con las avenidas torrenciales en los cauces, dos de las principales amenazas. La escasa información que se tiene de la precipitación muestra tres de los rasgos principales de la lluvia en Canarias: su acusada irregularidad interanual, su fuerte concentración en los meses de finales del otoño hasta principios de la primavera y su torrencialidad. Este último rasgo, junto con los importantes volúmenes de precipitación anual, explican la activación de diversos procesos geomorfológicos. En las últimas décadas, y a medida que hay una mayor afluencia de turistas, son significativas las noticias donde se refleja la evacuación de excursionistas que transitan por el Parque pues se ven sorprendidos por estos temporales destacando la muerte de tres de ellos el 20 de noviembre de 2001. La forma en que se produjo el siniestro demuestra cómo la precaución y la prevención son las mejores herramientas para evitar este tipo de sucesos. 


\section{REFERENCIAS}

Arozarena, A., Hernán, M., Vega J.A. (1976). Parque Nacional de la Caldera de Taburiente. Isla de La Palma. Madrid: Ministerio de Agricultura. Instituto Nacional para la Conservación de la Naturaleza.

Díez-Herrero, A., Génova, M., Mayer, P., Ballesteros, J.A., Becerril, L., Rubiales, J.M., Hernández-Ruiz, M., Saz, M.A., Bodoque, J.M., Ruiz-Villanueva, V. (2012). Investigaciones dendrogeomorfológicas aplicadas al estudio de la peligrosidad por avenidas e inundaciones en el Parque Nacional de la Caldera de Taburiente (La Palma, Islas Canarias). En A. González-Díez (coord.), XII Reunión Nacional de Geomorfología (pp. 159-162). Santander: PUbliCan Ediciones, Universidad de Cantabria.

Dorta, P. (2007). Catálogo de riesgos climáticos en Canarias: amenazas y vulnerabilidad. Geographicalia, 51, pp. 133-160.

Génova, M., Máyer, P., Ballesteros-Cánovas, J., Rubiales, J.M., Saz, M.A., DíezHerrero, A. (2015). Multidisciplinary study of flash floods in the Caldera de Taburiente National Park (Canary Islands, Spain). Catena 131, pp. 22-34.

Lorenzo, J.H. (2013). Lluvias torrenciales y desprendimientos en la cara Norte del Benjenado. Cambio en la geomorfología del barranco de Las Angustias. Informe técnico. La Palma: Parque Nacional de La Caldera de Taburiente.

Martín-Vide J. (2004). Spatial distribution of a daily precipitation concentration index in peninsular Spain. International Journal of Climatology, 24, pp. 959-971.

Marzol Ma.V., Yanes A., Romero C., Brito de Acevedo E., Prada S. y Martins A. (2006). Caractéristiques des précipitacions dans les îles de la Macaronesia (Les Açores, La Madère, Les Canaries et le Cap Vert). En G. Beltrando, M. Madelin y H. Quénol (Eds.), Les risques liés au temps et au climat (pp. 415-420). Maison Moët \& Chandon: Publications de l'A.I.C.

Rodríguez Fernández, R. (2011). Parque Nacional de la Caldera de Taburiente. Guía Geológica. León: Instituto Geológico y Minero de España y Organismo Autónomo de Parques Nacionales. 